\title{
"COMPETÊNCIA" NAS METODOLOGIAS ATIVAS E A IMPORTÂNCIA DE SEUS SIGNIFICADOS NA FORMAÇÃO DO ENGENHEIRO CIENTISTA
}

DOI: 10.37702/2175-957X.COBENGE.2021.3401

Rogério Bastos Quirino - rb_quirino@hotmail.com

UNEMAT

Av. das Embaúbas 567

78550-970 - Sinop - MT

Gilmar Barreto - gbarreto@dsif.fee.unicamp.br

Universidade Estadual de Campinas

Avenida Albert Einstein 400

13083-852 - Campinas - SP

Resumo: $O$ artigo discute o conceito de competências em educação em engenharia sob a ótica da formação do engenheiro com fundamentos e visão para a criação, domínio e desenvolvimento da ciência. Inegavelmente, existe um crescente aprofundamento no conceito de aprendizagem por competências em várias áreas da educação, formação e desenvolvimento profissional. Principalmente na aplicação das metodologias ativas, as competências são comumente consideradas para superar os níveis de conhecimento e habilidades, enquanto seu excedente é pensado para responder pela aplicação efetiva dos conhecimentos e habilidades disponíveis em um contexto específico. Infelizmente, junto com essa tendência, o termo "competência" tem sido utilizado de muitas maneiras diferentes na educação em engenharia, sem que nenhuma delas incorpore a formação do engenheiro com perfil científico. Como subsídio, utiliza-se uma abordagem de como as competências são pensadas para se relacionar com conhecimentos, habilidades e atitudes, e analisa o significado e a validade de uma das visões sobre competências. Nela, o termo tem significado adicional para o termo habilidades, o que é imprescindível à formação do engenheiro cientista. $O$ principal aspecto tratado neste artigo de formação do engenheiro cientista é de que há necessidade de se pensar numa "competência" no sentido de não necessariamente satisfazer uma expectativa de requisito. Além disso, o artigo trata da explicação teórica do termo, aqui compreendida e denominada como "competência acadêmica-científica". 


\section{(C) COBENGE

Palavras-chave: Ensino em Engenharia. Metodologias Ativas. Competências. Habilidades. Ciência e Tecnologia. 


\section{"COMPETÊNCIA" NAS METODOLOGIAS ATIVAS E A IMPORTÂNCIA DE SEUS SIGNIFICADOS NA FORMAÇÃO DO ENGENHEIRO CIENTISTA}

\section{INTRODUÇÃO}

As mudanças no campo profissional do "engenheiro" são evidentes, pois "O mundo está mudando rapidamente de uma organização nacionalmente diferenciada e identidades culturais para uma cada vez mais caracterizada por instituições transnacionais e comunidades multiculturais, acelerado por avanços tecnológicos dramáticos" (Continental AG, 2006).

A competição global impõe requisitos de alta qualidade aos produtos e serviços, aumenta a proximidade com o cliente e requer um nível de custo mais baixo. As empresas vêem potencial competitivo na redução do tempo de desenvolvimento de produtos para reduzir os custos do produto. "A resultante pressão para inovar garante que o processamento departamental rígido das tarefas de trabalho seja substituído pela cooperação dos departamentos individuais da empresa" (Continental, AG 2006; Völker, 2007).

Uma prática comum nas empresas de manufatura modernas é usar equipes multifuncionais para projetar e desenvolver novos produtos. Este processo, alternativamente referido para como desenvolvimento simultâneo, requer a integração de engenharia, marketing, finanças, e produção, a fim de minimizar o redesenho e retrabalho, assim, encurtando idealmente o ciclo do conceito à entrega" (Shirland \& Manock, 2000). "Esta dependência mútua de muitas etapas de desenvolvimento requer um maior grau de comunicação e esforço de coordenação. Lidar e trocar "conhecimento" passou a ter cada vez mais importância (Völker, 2007).

Procurando atender tais requisitos de inovação, segundo (Stephan Bode,2015, Tese Doutorado), quatro categorias de competência são exigidas à formação do engenheiro:

1. Competência técnica e metódica (conhecimento técnico e know-how em ciências naturais, ciências da engenharia, especialização em engenharia e capacidade de aplicação modernas tecnologias de informação e comunicação);

2. Competência pessoal (flexibilidade, formação geral profunda, disposição para realizar, disposição para se envolver na aprendizagem ao longo da vida, mobilidade, credibilidade e disposição para assumir responsabilidades);

3. Competência de gestão (qualificação gerencial, habilidades assertivas, capacidade de tomada de decisão, capacidade de analisar e avaliar, pensamento estratégico e habilidades de negociação);

4. Competência social (persistência, intuição, habilidades interculturais, capacidade de comunicação, capacidade de negociar compromissos e compensações e capacidade de trabalhar em equipes).

Entretanto, apesar da vasta literatura de educação em engenharia (Crawley, E. F. et al, 2014; Graham, R,2018; Balakrishnan, A. et al, 2008; Harwood, J.,2006; Feisel, L.D. \& Rosa, A.J., 2005; Rugarcia, A. et al, 2000; Subheesh, N.P., 2018) existente que se debruça nas preocupações com a formação e atuação científica e prática do engenheiro na sociedade, ainda existem resquícios de incorporação implícita de um entendimento dicotômico entre a educação, formação e desenvolvimento profissional do engenheiro e cientista pesquisador, que não contribui à reflexão de significados de competências ao consequente projeto de metodologias ativas a serem implementadas à educação em engenharia. 
A razão desta afirmação reside no fato de não se levar em conta no projeto das metodologias ativas, uma importantíssima categoria de competências imprescindível ao real desenvolvimento científico e tecnológico que somente é alcançado com a formação do engenheiro com o perfil de cientista, aqui denominada de competência acadêmicacientífica.

A formação do engenheiro deveria se coadunar com o significado de "competência" do vocabulário científico íntegro como sendo o uso de uma classe de sub-habilidades cognitivas que estão envolvidas no enfrentamento de problemas não padronizados e complexos, constituintes do cenário inerente da própria exploração técnica-científica, que não se enquadra em nenhuma das 4 categorias de competências anteriormente mencionadas, (Westera, W., 2001).

Naturalmente, o engenheiro deveria ser formado para o alcance do limiar da plenitude da visão técnica-científica dos sistemas em geral, que consiste na visão mor, macro, do todo, abstraída, criativa, inovadora, sem necessariamente ter "deadline" estabelecido para sua concretude com vista de fato a protagonismos e contribuições à ciência.

Com o intuito de preencher essa falta de entendimento e incorporação desse significado de "competência" no cenário das graduações em engenharia, caberia medidas prementes através das DCN's (Diretrizes Curriculares Nacionais) que concentrem esforços no sentido de que os cursos de engenharia internalizem o significado de competência analisado em (Westera,W.,2001), ao delineamento e proposição de metodologias ativas que em sua grande maioria ou totalidade, seja de mesma natureza promissora de educação, formação e desenvolvimento profissional do cientista, ainda não bem explorada nos cursos de engenharia.

Os níveis de abstração, criação, e protagonismo científicos de engenheiros e cientistas devem se equivaler, satisfazendo igualmente a validação de resultados e fins de aplicação como fim precípuo.

Tal medida contribuiria significativamente de forma não bifurcada e optativa, mas como caminho "sine qua non" à educação, formação e desenvolvimento profissional dos engenheiros, incorporando constituição científico-acadêmica de qualidade que não somente atenda às necessidades de inovação de mercado, mas também, ao prazer real de geração e domínio da ciência em benefício da sociedade.

Em (Robbins, J., 2015, pg. 146), uma coletânea de documentos que traz depoimentos de Richard P.Feynman registradas em suas palestras dirigidas ao público em geral e não aos seus colegas cientistas, Feynman em trecho abordando o prazer em descobrir as coisas e a valorização da ciência, diz:

"Outro valor da ciência é a diversão chamada prazer intelectual que alguns obtêm quando leem, aprendem e pensam sobre ela, e outros, quando trabalham com ela. Essa é uma questão muito real e importante que não é suficientemente levada em consideração pelos que nos dizem que nossa responsabilidade social é refletir sobre o impacto da ciência sobre a sociedade. Esse mero prazer pessoal tem valor para a sociedade como um todo? Não! Mas também há a responsabilidade de considerar o valor da própria sociedade. Em última análise, esse valor não seria organizar as coisas para que as pessoas pudessem ter prazer com elas? Se assim for, o prazer da ciência é tão importante quanto qualquer outra coisa." 
O 'Desvio Científico' na formação do engenheiro pode ser entendido como o processo pelo qual o conhecimento que se destina a ser "útil" perde gradualmente laços estreitos com a prática ao mesmo tempo que se torna mais fortemente integrado com um ou outro corpo de conhecimento científico.

Esse desvio de natureza acadêmica, neste sentido, foi um fenômeno comum na agricultura, ciências da engenharia, medicina e gestão em vários países no século 19 e Séculos 20, conforme estudo (Harwood, J., 2006), e que ocorre sem o devido tratamento ao seu aproveitamento até os dias de hoje.

Compreender e refletir sobre esse intervalo entre academia e atendimento ao mercado das aplicações práticas, é obviamente importante, ainda mais considerando que as metodologias ativas podem projetar ponderadamente as introjeções entre tais polos de desvio.

É surpreendente, portanto, que embora a discussão sobre a formação nos cursos de engenharia, focada nestes polos, tenha sido amplamente documentada, notavelmente pouco tem sido discutido sobre eles no contexto das atribuições de competências consideradas nas metodologias ativas.

Segundo (Harwood, J., 2010), o desvio não é uma tendência universal invariável, mas historicamente demonstra que surge em circunstâncias particulares.

Considerando as suas implicações nas metodologias do ensino superior, parece legítimo perguntar quais faculdades de engenharia se preocupam em refletir profundamente sobre a implementação de suas metodologias ativas no que tange as competências e habilidades que se adequam a seus graduandos para atuar, seja na indústria ou na academia.

O objetivo mais importante a ser alcançado com as técnicas de ensino é o de formar o graduando a fim de produzir conhecimento tanto técnico como científico para servir a humanidade. Essa posição, no entanto, significa que se deve rejeitar categoricamente o domínio de uma única polarização de desvio.

Em (Robbins, J., 2015, pg. 37), Richard P. Feynman abordando o ensino, declara:

"Todos os alunos estão na sala, e você me pergunta: como é o melhor jeito de dar aula pra eles? Eu deveria ensinar do ponto de vista da história da ciência ou das aplicações? Minha teoria é que a melhor maneira de ensinar é não ter filosofia nenhuma. Tem de ser caótico e confuso, no sentido de que a gente usa todos os jeitos possíveis de ensinar. Esse é o único jeito que consigo ver de responder essa pergunta, pra pegar esse cara ou aquele cara com ganchos diferentes pelo caminho, e na época em que o sujeito interessado em história fica de saco cheio da matemática abstrata, o sujeito que gosta das abstrações fica de saco cheio de história. Se a gente consegue ensinar de um jeito que não encha o saco de todo mundo o tempo todo, talvez seja melhor. Realmente não sei como é. Não sei responder a essa pergunta de tipos diferentes de cabeça com tipos diferentes de interesse: o que pega cada um, o que interessa a cada um, como levar todos eles a se interessar."

Igualmente, neste cenário de permeabilidade entre polos, a título de exemplo, Thomas Hughes, mostrou que apesar da importância da matemática na engenharia elétrica alemã do século 19 , engenheiros tiveram que vivenciar com a pouca utilidade dela na invenção de dispositivos de corrente contínua. 
Edison, por exemplo, tinha pouco conhecimento matemático, que era muito importante na construção e melhoria de equipamentos de corrente alternada (Hughes, 1983).

Da mesma forma, Edwin Layton (Layton, 1988) argumenta que o desenvolvimento de teorias de engenharia era sofisticado o suficiente para ajudar os projetistas a prever os efeitos de escala exigidos no uso de equações diferenciais.

Consequentemente, a maioria dos teóricos desta 'Revolução adimensional' eram engenheiros europeus bem versados no cálculo (como suas contrapartes americanas mais orientadas para a prática não eram.

O ponto chave é que não há um caminho certo para o sucesso na engenharia científica. $\mathrm{E}$ isso significa dizer que o planejamento das metodologias ativas deve se ater na importância da demonstração de existência de pontos quiescentes no domínio, aplicação e geração de conhecimento baseados na fronteira destes polos.

Deve-se preservar a diversidade de realidades de pessoas e situações, mas deve se insistir na formação de engenheiros que estejam preparados para o eficaz e rápido trânsito entre estes polos do universo de domínio técnico-científico.

É óbvio dizer que a história nunca se repete; o futuro nunca simplesmente reproduz o passado. Portanto, não é de se esperar que a história da educação do ensino de engenharia por si só forneça um plano para ação.

O que ela pode fazer, no entanto, é fornecer conjuntamente com a incorporação de estratégias de ensino-aprendizagem, ferramentas para reflexão, uma maneira de olhar para os problemas das dinâmicas de educação e desenvolvimento técnico-científico global e local atuais, de forma a antever e corrigir possíveis polarizações de desvios.

Muitas são as metodologias ativas existentes, as mais utilizadas são:

- Case Method Teaching (Aprendizagem por estudo de caso)

- PBL -Problem Based Learning (Aprendizagem Baseada em Problemas)

- Project-Based Learning (Aprendizagem Baseada em Projetos)

- Coached Problem Solving (Resolução de problemas por treinamento)

- Collaborative Learning (Aprendizagem colaborativa), G+Hangout sessions (Salas de discussão do $\mathrm{G}_{+}$)

- Guided Discovey Problems (Aprendizagem por descoberta Guiada), Just-in-Time (Resposta no tempo certo)

- Online Forum Discussion (Fórum Online de discussão)

- Remote Labs (Laboratórios Remotos), Retrieval Pratice (Prática da Recuperação da informação)

- Role-Playing (Aprendizagem por Interpretações de Papéis), Send-a-problem (Problema enviado)

- Team-Based Problem Solving (Solução de problemas em Equipes)

- Think-Pair-Shere (Pensar para compartilhar)

- Peer Instruction (Instrução entre os pares)

- Flipped Classroom (Sala de aula invertida)

entre outras, que podem ser aplicadas no ambiente de ensino, utilizando-se de exemplos de casos reais ou simulações para despertar o interesse no aluno.

Segundo (Cândido, J.,2019), oito são as estratégias possíveis de serem implementadas, através do uso das metodologias ativas em sala de aula: 
- Discussão de temas e tópicos de interesse profissional

- Trabalho em equipe com tarefas colaborativas

- Estudo de casos em áreas profissionais específicas

- Debates sobre temas da atualidade

- Geração de ideias para solução de um problema

- Uso de mapas mentais para aprofundar conceitos e ideias

- Modelagem e simulação de processos e sistemas

- Criação de espaços virtuais para aprendizagem coletiva

- Questões de pesquisa na área cientifica e tecnológica

As diferentes metodologias ativas aplicadas no ambiente de aula naturalmente promovem o envolvimento do aluno no processo de aprendizagem. Atualmente as tecnologias de comunicação e informação facilitam a busca de informações por parte dos alunos.

No entanto, implementar uma abordagem de aprendizagem ativa muitas vezes não é fácil e envolve a superação de inúmeras barreiras, muito em função do pouco espaço disponível nas matrizes curriculares que ainda mantêm as tradicionais estruturas pedagógicas, utilizando-se de aulas expositivas e, ainda é possível encontrar a divisão de conteúdo em disciplinas subsequentes o que não corrobora para o desenvolvimento e aplicação dessas metodologias, (CÂNDIDO, J., 2019).

Além disso, a aprendizagem ativa muitas vezes requer mais tempo e sacrifício de cobertura do conteúdo do curso em sala de aula. Ao rompimento de barreiras, recomendase que os educadores implementem aprendizagem ativa em seus cursos adicionando conteúdo por meio do site do curso, com a utilização de artigos específicos e questionários para garantir a conclusão de leituras, cursos online, mini-palestras, pré-aulas, ou mesmo palestras completas online.

Outras barreiras dizem respeito ao tempo envolvido na criação tarefas de aprendizagem ativa, riscos envolvidos na implementação e cultura institucional ou falta de "adesão" à aprendizagem ativa.

A quantidade de tempo necessária para conceber os componentes específicos da aprendizagem ativa podem envolver um tempo de preparação semelhante ao necessário para a criação de uma nova aula. Alguns professores podem não ter confiança para implementar a aprendizagem ativa ou podem achar que é arriscado compartilhar controle da sessão de aula com os alunos.

Outras barreiras potenciais incluem a falta de interesse do aluno, vontade ou preparação para participar de atividades aprendendo.

Dar aos alunos expectativas claras e instruções sobre como participar neste tipo de experiência de aprendizagem em sala de aula pode ser útil.

Os alunos podem aprender conceitos básicos do curso por meio da preparação da pré-aula, como tarefas de leitura, os instrutores não devem usar o tempo das aulas para dar uma palestra sobre esses conceitos básicos, mas antes deveria seja franco e ajude os alunos a entender o fundamento lógico para implementar a aprendizagem ativa.

Para alunos que são novos para esta abordagem de aprendizagem em sala de aula, o instrutor deve garantir que a participação inicial seja divertida, por exemplo, por meio do uso de um programa de aprendizado ativo baseado em jogos.

Criando um ambiente adequado no qual os alunos podem aprender, pensar, ser avaliado e receber feedback é importante. 
Finalmente, os instrutores devem buscar maneiras de motivar participação do aluno, como a atribuição de pontos de participação ou promovendo competição amigável. Por exemplo, o grupo que "ganhe" o jogo pode receber algum tipo de prêmio.

Os alunos podem resistir ao aprendizado ativo no início, mas dando-lhes uma compreensão clara do propósito dessas atividades e responsabilizá-los pela participação pode melhorar sua participação nas aulas e seu desempenho no curso avaliações. Como resultado, os alunos podem vir a se tornarem mais animados e motivados para aprender.

Entretanto, na exploração da gama de caminhos alternativos disponíveis à implementação das metodologias ativas, ainda persiste a necessidade sempre premente de se fazer a devida internalização do conceito científico rigorosamente crítico de "competência" incorporando-o como o objetivo central a ser alcançado por meio das estratégias de implementação das metodologias ativas, a fim de subsidiar e prover meios para que os engenheiros adquiram formação com perfil de cientista, (Westera, W., 2001).

Vale ressaltar que nas estratégias de implementação supracitadas de metodologias ativas, a que propõe abordar "Questões de pesquisa na área cientifica e tecnológica", apresenta se mais explicitamente à incorporação desse novo conceito de "competência".

Ademais, pode se admitir que um bom desenvolvimento dessa estratégia baseado neste novo conceito e num estudo aprofundado e detalhado da história da ciência e tecnologia, alicerça em escopo e filosofia o bom desenvolvimento de grande parte das estratégias supracitadas restantes, pois implícita ou explicitamente, com menor ou maior grau, em contextos e realidades diferentes, elas já foram inexorável e historicamente realizadas.

O plano base de esculturação do conhecimento prévio em ciência e tecnologia e do significado de competência utilizados nessa abordagem sobre questões de pesquisa na área científica e tecnológica pode conduzir a desvios de educação em engenharia indesejados na medida em que, sem o estabelecimento de critérios importantes, pode vir a utilizar ferramentas de mera satisfação ao atendimento de formação superficial, por exemplo, à demanda dos sistemas 4.0, (Quirino et al, 2020).

O conceito de competência é bastante abrangente. Foi apresentado por muitos autores como uma nova entidade para explicar certos comportamentos em certas situações.

No entanto, a lei causal que se relaciona competência para desempenho competente, representa uma proposição tautológica que ainda merece explicações.

Portanto, a competência não pode ser entendido com significados padrões, que não aumentam nosso conhecimento e compreensão do mundo, exatamente o oposto do esperado na formação científica.

Por definição, as competências deveriam estar associadas a comportamentos de sucesso em situações não padronizadas, exigidas na formação do engenheiro cientista, apesar de tal definição parecer entrar em conflito com o uso de competências como padrões educacionais.

Consequentemente, as atuais avaliações de competências e seus valores preditivos para desempenhos futuros são altamente questionáveis quando utilizadas na formação do engenheiro que deve ter sólida formação à exploração científica. As avaliações de competências pensadas na atualidade não deveriam necessariamente lidar com a reprodutibilidade ou, melhor, com a previsão de comportamentos futuros de sucesso, que inerentemente não obedece a dinâmica do processo científico.

Isso também se relaciona com a suposta instabilidade do conceito de competência e sua forte dependência de valor.

Em coerência com estabilidade dos valores, as competências podem facilmente tornar-se objeto de mudança. 
Em retrospecto, gerentes, políticos ou cientistas animados e "competentes" são facilmente condenados, quando os efeitos de suas ações parecem estar em contraste com promessas ou buscas.

Os políticos geralmente tendem a se empenhar por sucessos de curto prazo para ganhar popularidade e os empresários à liquidez financeira dos produtos e sistemas com as tecnologias vigentes embarcadas .

No que diz respeito às diferenças entre competências e habilidades, o pensamento consciente não pode servir como um fator discriminativo, uma vez que naturalmente, a complexidade e a novidade de uma situação parecem provocar o comportamento consciente. Este pensamento consciente diz respeito à seleção, combinação ou adaptação de rotinas existentes para atender a situação.

Além disso, a idéia de complexidade parece indicar que as competências são uma subclasse de habilidades, e não uma categoria de coordenação.

As habilidades são rotinas mais ou menos automatizadas que permitem realizar tarefas bem específicas. Isso não significa necessariamente que as habilidades sejam simples e diretas. Por exemplo, pilotar um avião exigiria habilidades sofisticadas. Conforme o nível de complexidade aumenta, a mera presença de habilidades deve ser insuficiente para explicar o comportamento de sucesso. Provavelmente, em más condições climáticas, pode-se preferir um piloto competente a um experiente. Isso levanta a questão de como o nível de complexidade é descrito para discriminar entre competências e habilidades, (Kirby,1988).

Em teoria, a complexidade de uma determinada tarefa pode ser determinada a partir do número de objetos envolvidos, seus atributos, suas inter-relações, as regras e processos envolvidos e assim por diante. Mas, além do trabalho árduo, a complexidade de uma tarefa deve sempre ser avaliada em relação a pessoa envolvida.

Portanto, pode-se concluir que existem dois problemas principais com o conceito de competência a serem considerados na formação do engenheiro cientista. $O$ primeiro, que o conceito de competência tenta definir padrões cognitivos para comportamentos que essencialmente não podem ser padronizados. O segundo, do ponto de vista científico, as competências devem ser consideradas para compor uma subcategoria de Habilidades.

Sinteticamente, as metodologias ativas deveriam tomar como base os modelos de competência explorados em (Westera, W. 2001).

O modelo da "Figura 1" ao invés do modelo padrão da "Figura 2", demonstra ser mais adequado à formação do engenheiro cientista, em razão de propiciar a exploração relevante dos infinitos estágios entre academia e prática na implementação das aprendizagens ativas como inspiração humana ao bem estar mental do estudante de engenharia, gerado pela real capacidade de abstração de conhecimentos e de justiça na ciência e engenharia, apoiada no possuir (conhecimento), sentimento (atitudes), fazer (habilidades), criar(engenheiro) e descobrir(cientista), suplantando um histórico de ensino de engenharia marcado pelo mero acompanhamento marginal, robótico, empacotado, não processado, sem protagonismo, do desenvolvimento científico e tecnológico mundial. 
Figura 1 - Competências como Sub-Habilidades

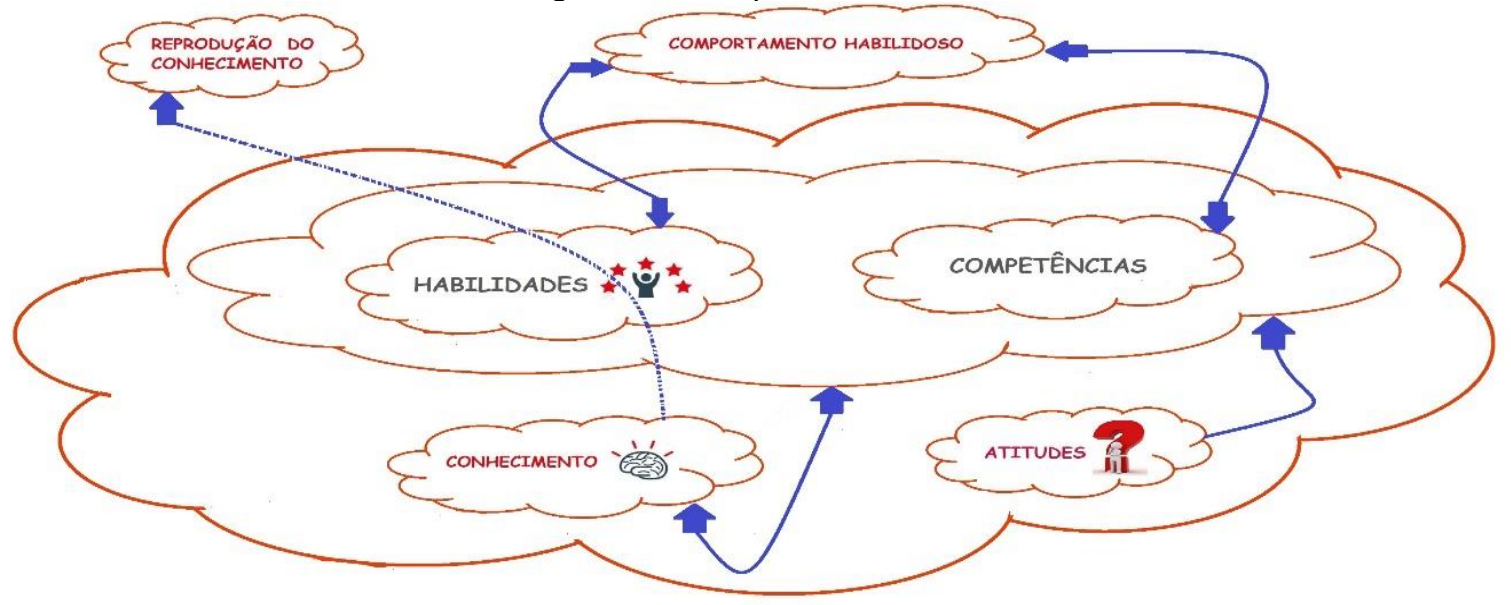

Fonte: Autores

No entanto cabe ressaltar que a remoção de competências como uma categoria distinta de habilidades humanas, destacada na Figura 1, não significa que o termo deve ser banido. Não pode haver objeções ao uso do termo competência como um rótulo.

Provavelmente, o termo pode ser usado com sucesso para o público para fins de relacionamento, em razão de suas principais associações com qualidade e expertise. Da mesma forma, o termo competência pode ser reservado para indicar que a associação de conhecimentos e habilidades origina-se da prática profissional.

Além disso, de um ponto de vista científico, o termo competências é muito problemático e carece de uma definição comum e sensata. Possivelmente, seu uso deve ser restrito a uma classe de sub-habilidades cognitivas que estão envolvidas no enfrentamento de problemas complexos.

Embora os resultados desta análise possam ser decepcionantes para qualquer pessoa preocupada com aprendizagem de competências, a remoção da categoria distinta de competências parece ser a única maneira de preservar um vocabulário científico íntegro e incorporá-lo na formação do engenheiro cientista.

Figura 2 - Um modelo de competência de acordo com definições padrão.

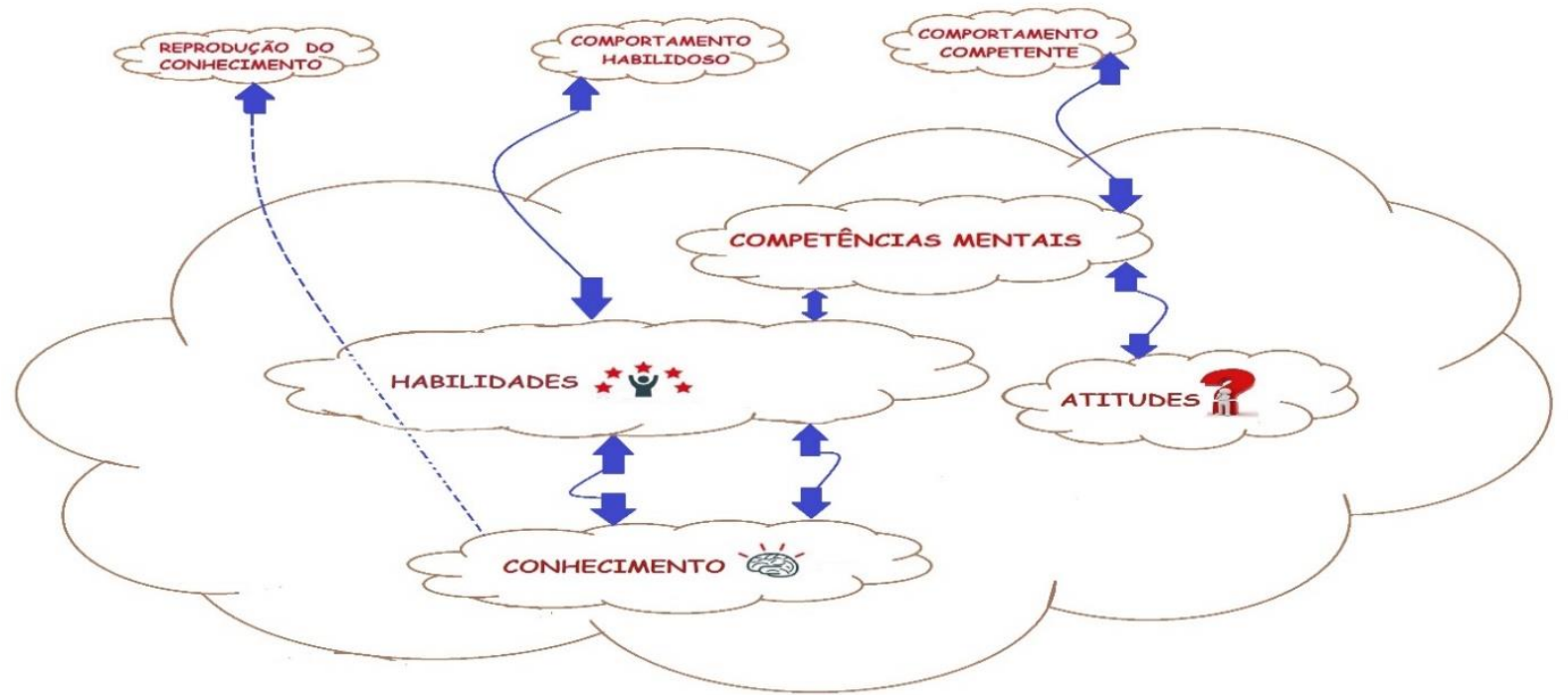

Fonte: Autores 


\section{CONSIDERAÇÕES FINAIS}

As competências na formação do engenheiro cientista, por definição, deveria estar associadas a comportamentos de sucesso em situações não padronizadas. Tal definição apesar de entrar em conflito com o uso de competências como padrões educacionais, elas devem servir de balizamento para que os estudantes de graduação de engenharia obtenham resultados realmente significativos para sua formação compatível com a de cientistas.

O uso de competências como padrões educacionais não deveria se referir necessária e exclusivamente às tarefas envolvidas em si mesmas, mas serem associadas em primeiro plano, com a insistente componente de despertar, instigar e preparar os estudantes ao trânsito maduro nessa fronteira da ciência e tecnologia, quaisquer que sejam as características e formação prévias desses estudantes. Claramente, isso requer o uso de um novo significado de competência dissociado do significado de referência utilizado nos sistemas educacionais.

No processo de formação do engenheiro cientista, é importante levar em consideração três importantes dimensões orientadoras do conhecimento e habilidades: (1) a nomenclatura puramente declarativa de um comportamento específico no contexto da história da ciência e tecnologia; (2) a disposição individual com a qual os acadêmicos moldam sua vida na graduação que geram motivações e desenvolvam metas de ação; e (3) o cenário de possibilidades sob as quais as disposições dos acadêmicos se desenvolvem e aí obtêm requisitos.

Em suma, quais fatores nas metodologias ativas vigentes tendem a estimular única e exclusivamente o alcance e a permanência num dos lados da fronteira da ciência e tecnologia e quais conduzem para o inteligente, deslumbrante, prazeroso permear sobre ela? O lugar natural para as respostas seria desenvolver um estudo sistêmico recorrente sobre a história da engenharia, ciência e tecnologia, e sua relação permanente e significativa com as competências ativas imbricadas na filosofia e psicologia da educação em sentido mais estrito (STROET, K. 2015), a fim de se estabelecer as complementaridades e dar continuidade cada vez mais impreterível ao entendimento e traçado de estratégias de metodologias ativas de ensino de engenharia com o passar do tempo.

Finalmente, desejar que a abstração de qualificação das competências à aplicação nas metodologias ativas realizada neste trabalho frente aos desafios reais de eficácia e relevância de desenvolvimento científico e tecnológico sirva de inspiração e ferramenta ao desenvolvimento de estudos que parametrizem e quantifiquem as metodologias ativas no ensino de engenharia com o propósito de criar modelos de mérito à análise e ajustes das práticas de ensino do engenheiro pensadas que incorporem a visão de produção de ciência, lembrando que a ciência, dentre todas as matérias, contém dentro de si a lição do perigo da crença na infalibilidade dos maiores professores da geração anterior.

\section{REFERÊNCIAS}

BALAKRISHNAN, A.; LAL, B.; FLATTAU, P.E. The Evaluation of Engineering Education Research: Emerging Issues and Promising Developments. Workshop Report Ida Institute For Defense Analyses Science \& Technology Policy Institute, October. Washington, DC, USA. 2008. 
Bode, S. "Kompetenzverhandlungen"- Zur Relevanz von Sozialkompetenz bei Führungskräften der Continental AG am Beispiel dual Studierender Theoretische Grundlagen und Empirische Befunde. Tese (Doutorado) - Curso de Filosofia da Universidade de Hannover, Alemanha, 2015. Disponível em:. https://www.repo.unihannover.de/bitstream/handle/123456789/8468/828612056.pdf?sequence=1\&isAllowed=y Acesso em:23/04/2021.

CÂNDIDO, J. Contribuições para a Formação Docente em Cursos de Engenharia: Sua Importância e Necessidade. Tese de Doutorado, FEEC, Unicamp, 2019.

Continental AG. In search of global engineering excellence - Educating the next generation of engineers for the global workplace. Hannover: Münstermann, 2006.

CRAWLEY, E. F et al. Rethinking Engineering Education. The CDIO Approach. $2^{\text {nd }}$ Edition. Editora Springer, 2014.

CRUZ, Mariana Leandro; SAUNDERS-SMITS, Gillian N; GROEN, Pim. Evaluation of competency methods in engineering education: a systematic review. European Journal of Engineering Education, 2019.

FEISEL, L.D.; ROSA, A.J. The Role of the Laboratory in Undergraduate Engineering Education. Journal of Engineering Education. January, 2005.

GONZÁlEZ-MARCOS, A.; ALBA-ELÍAS, F.; ORDIERES-MERÉ, J. An Analytical Method for Measuring Competence in Project Management. British Journal of Educational Technology, 2015.

GRAHAM, R. The global state of the art in engineering education. School of Engineering, MIT, Cambridge, USA. 2018.

HARWOOD, J. Engineering Education between Science and Practice: The Historiography. History and Technology, Taylor \& Francis, March 2006, v. 22, n. 1, p. 53-79.

HARWOOD, J. Understanding Academic Drift: On the Institutional Dynamics of Higher Technical and Professional Education. Minerva - A Review of Science, Learning and Policy, v. 48, n. 4, 2010.

KIRBY, J.R. Style, Strategy and Skill in Reading. In: R.R. Schmeck (ed.) Learning Strategies and Learning Styles. Plenum Press, New York, 1988, p. 229-274.

LAYTON, E. American Institute of Physics In the Michelson Era in American Science. The dimensional evolution: the new relations between theory and experiment in 
engineering in the age of Michelson, 1870-1930, Ed. S. Goldberg, and R. Stuewer, New York, 1988. p. 23-41.

QUIRINO, R. B.; BARRETO, G.; CAMARGO, J.T.F. Preocupações Inerentes na Aplicação das Metodologias Ativas nas Graduações em Engenharia, Congresso Brasileiro de Educação em Engenharia UCS, Anais Caxias-RS, 2020. Disponível em: http://www.abenge.org.br/sis submetidos.php?acao=abrir\&evento=COBENG E20\&codigo=COBENGE20 00127 00003191.pdf . Acesso em: 09 dezembro 2020.

ROBBINS, J. Os Melhores Textos de Richard P. Feynman. Editora Blucher, 2015.

Shirland, L. E./ Manock, J. C.. Collaborative teaching of integrated product development: a case study“. IEEE Transactions On Education. 43(3): 343-348. 2000.

STROET, K.; OPDENAKKER, M.C; MINNAERT, A. Need supportive teaching in practice: a narrative analysis in schools with contrasting educational approaches. Social Psychology Education Journal, 2015.

VÖLKER, N. Neue Anforderungsprofile für Ingenieure - Kompetenzentwicklung durch Kooperation von Universität und Unternehmen. Tese (Doutorado) - Universidade de Tecnologia de Darmstadt, Alemanha, 2007. Disponível em: https://www.shaker.eu/en/content/catalogue/index.asp?lang=en\&ID=4\&category=236\&ag= 40. Acesso em: 23 abril de 2021.

WESTERA, W. Competences in education: a confusion of tongues. Journal of Curriculum Studies Research, 33 (1), p. 75-88, 2001.

\section{“COMPETENCE” IN ACTIVE METHODOLOGIES AND THE IMPORTANCE OF ITS MEANINGS IN THE FORMATION OF SCIENTIST ENGINEER}

Abstract: This article discusses the concept of competence in engineering education from the perspective of the training of engineers with fundamentals and vision for the creation, mastery and development of science. Undeniably, there is an increasing deepening in the concept of competency learning in various areas of education, training and professional development. Especially in the application of active methodologies, competencies are commonly considered to overcome levels of knowledge and skills, while their surplus is thought to account for the effective application of the knowledge and skills available in a specific context. Unfortunately, along with this trend, the term "competence" has been used in many different ways in engineering education, without any of them incorporating the training of an engineer with a scientific profile. As a subsidy, an approach is used on how competencies are thought to relate to knowledge, skills and attitudes, and analyzes the meaning and validity of one of the views on competencies. In it, the term has additional meaning for the term skills, which is essential to the training of the scientist engineer. The main aspect dealt with in this article for the training of the scientist engineer is that there is a need to think about a "competence" in the sense of not necessarily satisfying a requirement 
expectation. In addition, the article deals with the theoretical explanation of the term, here understood and named as "academic-scientific competence".

Keywords: Engineering Education. Engineering Teaching. Active Methodologies. Competences. Skills. Science and Technology. 